\title{
Traffic aggregation nodes placement for virtual EPC
}

\author{
Mariia Skulysh, Svitlana Sulima \\ Institute of telecommunication systems \\ National Technical University of Ukraine "Igor Sikorsky \\ Kyiv Polytechnic Institute" \\ Kyiv, Ukraine \\ itssulima@gmail.com
}

\author{
Ganna Grynkevych \\ Department of Telecommunication systems and networks \\ State University of Telecommunications \\ Kyiv, Ukraine
}

\begin{abstract}
The softwarization in the field of providing mobile communication services is outlined. The method of determining the binding of base stations to traffic aggregation points and their quantity in LTE EPC networks built based on NFV technology is proposed, which allows solving the problem of calculating the topology of EPC as a service, while taking into account the cost of organizing the service environment with minimizing delay and rational use of channels throughput capacity.
\end{abstract}

Keywords—NFV; EPC; node placement; resource allocation

\section{INTRODUCTION}

Mobile networks play an extremely important role in the global society informatization. With the growth of mobile data traffic and the number of services provided by the mobile network, signaling flows are also increasing, which necessitates changes in the principles, models and methods of organizing telecommunication service maintenance in order to ensure the given quality of providing a wide range of services in a flexible and cost-effective manner. Provision of services in the telecommunication industry is traditionally based on the fact that network operators implement static physical equipment for each network function that is located at fixed points and has fixed service characteristics.

Future generations' networks in comparison with previous systems, pose new challenges and requirements in terms of network flexibility and scalability, as well as increased demands for resource efficiency and associated economic profitability. With the development of infocommunication technologies, the dedicated hardware is rapidly becoming outdated and becomes the main area of expenditure for modern communication networks. Moreover, the irregularity in the load of infrastructure nodes leads to their underload, thus there is a need for the introduction of technologies that reduce equipment underload and guarantee Quality of Service at a given level throughout the day.

To meet these challenging goals, network softwarization is envisaged as the cornerstone to build such networks [1], [2]. The availability of high-capacity data centers greatly expands the possibilities of organizing the process of providing services in the modern telecommunications market. Telecommunication providers are increasingly using Network Function Virtualization (NFV) technology to rebuild their network as NFV infrastructure with data centers that can dynamically and flexibly build up service resources to provide services with a given quality level anywhere and anytime. Such NFV datacenters consolidate network appliance functions onto commodity servers and devices, which helps service providers to speed service deployment and reduce cost. NFV datacenters can be directed by programmable control planes with the Software Defined Networking (SDN) protocol to intelligently steer user traffic flows to the best suited network function unit therein [3].

So the concept of network softwarization is mainly based on i) NFV, which decouples network functions from proprietary hardware enabling them to run as software on virtualization containers such as virtual machines [4], and ii) SDN, which fully separates control and data planes in network nodes allowing network programmability [2].

Some potential use cases in the Telecom sector [5] are the ease of deployment of nodes like MME, S/P-GW [6], ondemand scaling of such nodes based on instant load rather than peak load based over-provisioning [7], dynamic topology reconfiguration for disaster avoidance and recovery [8], etc.

One of the key aspects of network virtualization is the distribution of physical resources for virtual network functions, which involves mapping virtual networks to physical networks. To organize such a computing-telecommunication environment, it is necessary to initially perform the initial reservation of network functions resources before system operation starts. The optimality and flexibility of resource allocation are key factors for successful virtualization.

Let's assume that the definition of the location and the required capacity of the reserved virtual computing resources in the event of an overload of the physical network is carried out in accordance with [9]. Let's develop this method.

The number of service chains must be determined in advance [10]. The extreme case would be consideration of one service chain per cell/eNodeB. Since realistic scenarios for mobile networks contain 10000 eNodeB, the resulting optimization model will be enormous, and a long enough computation time is required to solve it. Therefore, we accept reasonably large clusters of eNodeBs and assume that each of these clusters refers to a single service chain of the core network. 
Most modern research does not consider the task of determining the number of Evolved Packet Core (EPC) networks as services, and presupposes their predetermined amount. The closest is the research task from [11], which, however, seeks to determine the placement and binding of RRH sites to BBU servers in networks using NFV technology and does not take into account delay and bandwidth constraints.

Next we describe the algorithm for traffic aggregation nodes selection. In particular, we consider the case where the provider of telecommunication services already has an existing topology of base stations. It is needed to define a subset of the network nodes where traffic aggregation points will be placed, which will generate requests to one virtualized EPC service. After that, for each base station site, we assign a node of aggregation (Traffic Aggregation Point - TAP).

\section{PROBLEM FORMULATION FOR TRAFFIC AGGREGATION POINTS SELECTING}

The problem is illustrated in Fig. 1. In this figure, a certain number of base stations is grouped and assigned to one TAP.

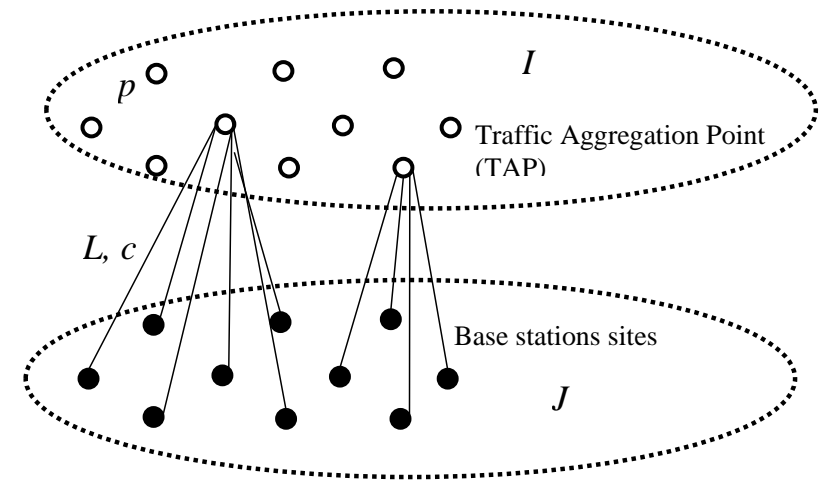

Fig. 1. Aggregation node allocation problem

\section{A. eNodeB sites}

We consider a network with a set $J$ of eNodeB sites, which should be serviced by another set $I$ of aggregation nodes, where $|I| \leq|J|$. The location of each site $j \in J$ is characterized by geographical latitude and longitude. Each base station site $j \in J$ provides services to subscribers whose combined average processing requirements are $d_{j}$. For each site, the maximum delay level $T_{j}$ is defined, which specifies the maximum distance from the site to the TAP.

\section{B. TAP}

We assume that the provider can set a maximum $p=$ $|I| \leq|J|$ of physical TAP to service all radio access network sites. Each TAP $i \in I$ has the maximum processing capacity $P_{i}$. This maximum computing capacity is intended to reflect the fact that in practice it is impossible to have a TAP with unlimited computing resources. The placement of TAP $i \in I$ includes the cost cost $_{i}$ which can be defined as a linear function of value, shown in (1). The cost is comprised of two parts: the fixed initial cost $f_{i}$, which is responsible for fixed investments such as space and equipment installation, as well as the additional $\operatorname{cost} \operatorname{cost} N_{i}$ per unit of processing capacity installed on the computing node, where $d r_{i}$ is the amount of processing resources involved.

$$
\operatorname{cost}_{i}=f_{i}+\operatorname{cost} N_{i} \cdot d r_{i}
$$

\section{Communication channels}

Each site is connected to the corresponding TAP via the communication channel $e_{j i} \in E$, where $E$ is a set of all communication channels. Each link $e_{j i}$ has $L_{j i}$ delay, which depends on the distance between the site $j$ and TAP $i$ and the signal speed in the transport environment used, as well as the available bandwidth $c_{j i}$. The cost of establishing a connection between the site $\mathrm{j}$ and TAP $\mathrm{i}$ is equal to $\operatorname{costl}_{j i}$ and is defined as the linear combination of the initial fixed cost $f l_{j i}$ and the variable part dependent on the required bandwidth $B_{j i}$ and the bandwidth cost $\cos t L_{j i}$, as shown in (2).

$$
\operatorname{cost} l_{i}=f l_{i}+\operatorname{cost} L_{i j} \cdot B_{i j}
$$

\section{OPTIMIZATION TASK STATEMENT}

\section{A. Definition of decision variables}

Let $x_{i}$ be a binary decision variable that takes value 1 if TAP is placed at point $i$, and 0 otherwise. In addition, we define $y_{j i}$ as a binary variable that takes value 1 if the base station $j$ directs the load to the $i$-th TAP and 0 otherwise. To find the optimal value of the objective function You need to define the values of $x_{i}$ and $y_{j i}$.

\section{B. Optimization goals} latency.

The objective function (3) seeks to minimize network

Objective function (4) represents the total cost of aggregation nodes establishing and the cost of establishing channels between the base stations and the respective TAPs that serve them. With:

$$
\operatorname{cost}_{i}=f_{i}+\operatorname{cost} N_{i} \cdot d r_{i}=f_{i}+\cos t N_{i} \cdot \sum_{j} y_{j i} \cdot d_{j}
$$

Objective (5) aims to leave more free bandwidth on each physical channel. The residual bandwidth of all channels is maximized, since highly-downloaded channels can lead to network overload, so it is advisable to get a solution where more channel capacity is left.

These optimization goals can be useful for network operators to plan the best deployment strategy.

$$
\min _{y_{j i}}\left(\sum_{i} \sum_{j} y_{j i} \cdot L_{j i}\right)
$$




$$
\begin{gathered}
\min _{x_{i}, y_{j i}}\left(\sum_{i} x_{i} \cdot \cos _{i}+\sum_{i} \sum_{j} y_{j i} \cdot \operatorname{cost}_{j i}\right) \\
\max _{x_{i}, y_{j i}}\left(\sum_{i} \sum_{j} y_{j i} \cdot\left(c_{j i}-B_{j i}\right)\right)
\end{gathered}
$$

We can use the linear combination (6) of expressions (3)-(5) with weighting factors $a, b, c$, which can be used not only to give greater weight to certain component, but also to scale the expression values for the purpose of balancing to comparable values and having a meaningful summation.

$\min _{x_{i}, y_{j i}}\left(\begin{array}{c}a \cdot \sum_{i} \sum_{j} y_{j i} \cdot L_{j i}+b \cdot\left(\sum_{i} x_{i} \cdot \operatorname{cost} N_{i}+\sum_{i} \sum_{j} y_{j i} \cdot \cos t L_{j i}\right)- \\ -c \cdot\left(\sum_{i} \sum_{j} y_{j i} \cdot\left(c_{j i}-B_{j i}\right)\right)\end{array}\right)$

\section{Constraints}

Constraint (7) ensures that each base station is connected to one TAP. Constraint (8) ensures that a channel is created between the base station site $j$ and the TAP $i$ only if $i$ was placed. Together, the constraints (7) and (8) ensure that the required number of TAPs is placed to serve all the sites, and base stations are assigned only to the nodes where the TAP is located.

$$
\begin{aligned}
& \sum_{i} y_{j i}=1 \forall j \\
& y_{j i} \leq x_{i} \forall j \forall i
\end{aligned}
$$

The constraint (9) ensures that the maximum number of TAP does not exceed the budget $p r$, while (10) is capacity limitation that ensures that the general processing requirements for all base stations assigned to a particular TAP do not exceed the actual physical resources installed. Constraint (11) guarantees the sufficiency of channel resources for the channels establishment, and (12) is the admissibility of the delay value constraint.

$$
\sum_{i} x_{i} \leq p r
$$

$$
\sum_{j} y_{j i} \cdot d_{j} \leq p_{i} \forall i
$$

$$
\sum_{i} y_{j i} \cdot\left(c_{j i}-B_{j i}\right) \geq 0 \forall j
$$

$$
\sum_{i} y_{j i} \cdot L_{j i} \leq T_{j} \forall j
$$

Solving of the problem (1)-(12) can be provided with the use of heuristic optimization methods (for example, genetic algorithm, etc.).

After that, it is proposed to apply the method of binding each network function of the traditional network to the data center and the amount of resources allocated to the corresponding virtualized network function, such that the objective function of the total cost of the leased resources is minimized, as described in [9], while providing an acceptable latency for service requests. A mathematical problem of nonlinear optimization is solved, which takes into account the available resources at the nodes and their cost, the intensity of excessive service load and allowable delays.

\section{SIMULATION RESUlTS}

The estimation of the operation of the simplified model (6)-(12) was performed in the Matlab simulation environment and the approach was compared with delay minimization approach. The model of the system with 10 nodes and 3 maximum possible aggregation nodes was considered, network parameters and resources cost were generated randomly. An example of solving the problem of aggregation points placing according to the proposed method and the least delay method is illustrated in Fig. 2.

The results of experiments showed that it is possible to reduce costs twice, but with the price of a corresponding increase in the delay.

A fragment of the code is shown in Fig. 3.

\section{CONCLUSIONS}

The prospective areas of research in the field of providing mobile communication services are outlined. The method of determining the binding of base stations to the traffic aggregation nodes and their amount in virtualized networks is proposed, which allows solving the problem of calculating the topology of EPC as a service, while taking into account the cost of organizing the infocommunication environment with minimizing delay and rational use of bandwidth. 


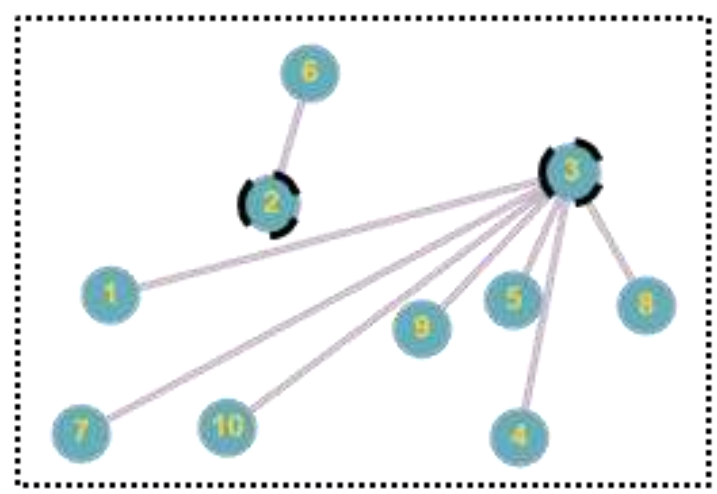

(a)

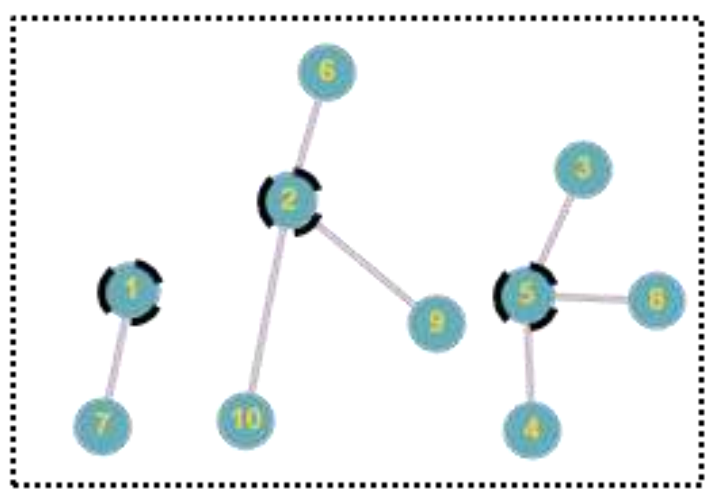

(b)

Fig. 2. Traffic aggregation node placement according to the proposed method (a) and least delay method (b)

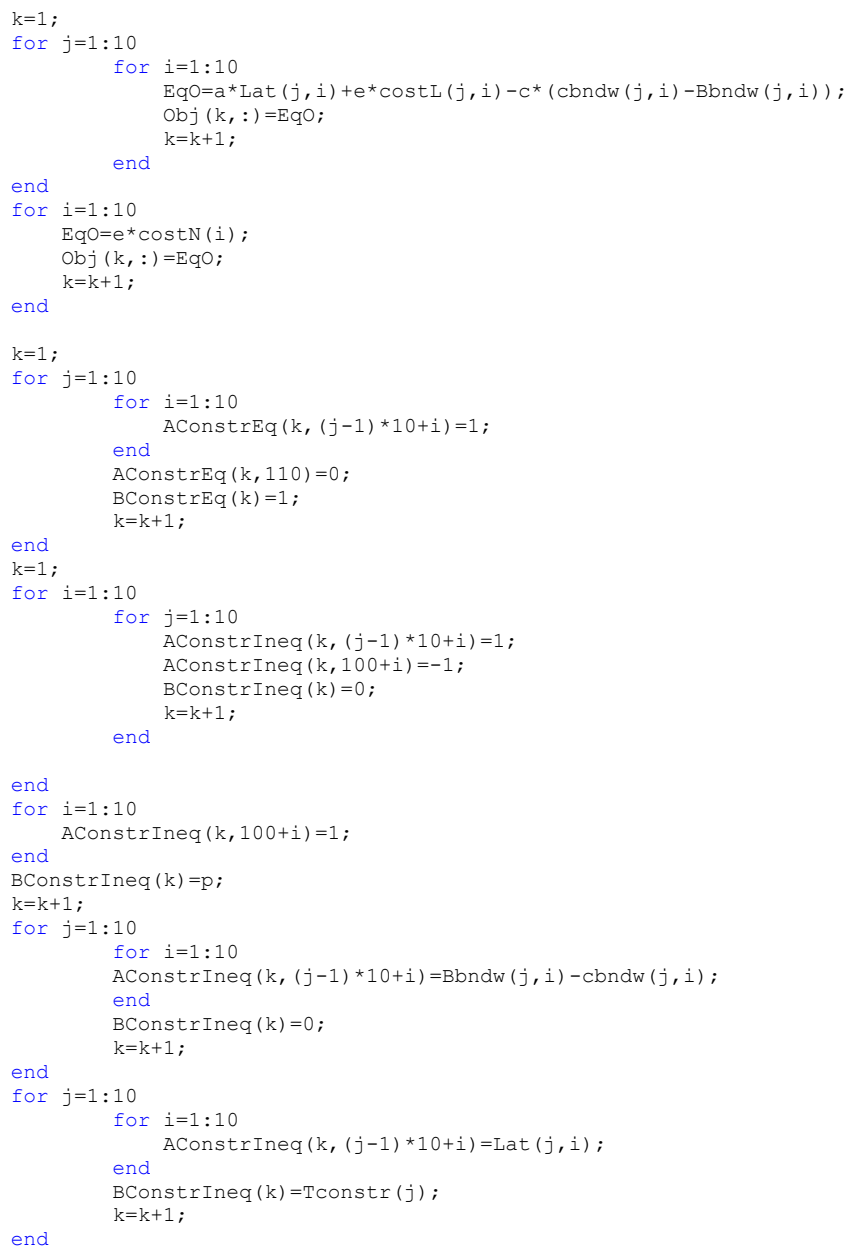

Fig. 3. Placement problem program code fragment

\section{REFERENCES}

[1] 3GPP TR23.799 V14.0.0. (2016) Study on Architecture for Next Generation System.

[2] J. Prados-Garzon, A. Laghrissi, M. Bagaa, T. Taleb, and J.M. LopezSoler, "A Complete LTE Mathematical Framework for the Network Slice Planning of the EPC," IEEE Transactions on Mobile Computing ( Early Access ), pp. 1-14, January 2019.

[3] Y. Lin, C. Wang, C. Huang, and Y. Lai, "Hierarchical CORD for NFV Datacenters: Resource Allocation with Cost-Latency Tradeoff," IEEE Network, vol. 32, pp. 124-130 April 2018.

[4] H. Hawilo, A. Shami, M. Mirahmadi, and R. Asal, "Nfv: state of the art, challenges, and implementation in next generation mobile networks (vepc)," IEEE Network, vol. 28, no. 6, pp. 18-26, Nov 2014.

[5] A. Khan, X. An, and S. Iwashina, "Virtual Network Embedding for telco-grade network protection and service availability," Computer Communications, vol. 84, pp. 25-38, June 2016.

[6] 3GPP TS 23.401, General Packet Radio Service (GPRS) enhancements for Evolved Universal Terrest rial Radio Access Network (E-UTRAN) access, Technical Specification Group Services and System Aspects, 3rd Generation Partnership Project, verion 13.3.0.

[7] Y. Takano, A. Khan, M. Tamura, S. Iwashina, T. Shimizu, "Virtualization-based scaling methods for stateful cellular network nodes using elastic core architecture," in: Proceedings of the 6th IEEE International Conference on Cloud Computing Technology and Science (CloudCom'14), December 2014.

[8] T. Shimizu, T. Nakamura, M. Kiuchi, A. Iwata, Y. Kubota, M. Ohhashi, "An experimental evaluation of dynamic virtualized networking resource control on an evolved mobile core network," in: Proceedings of the Humanitarian Technology Conference (HTC'13), August 2013.

[9] L. Globa, M. Skulysh, S. Sulima, "Method for resource allocation of virtualized network functions in hybrid environment," 2016 IEEE International Black Sea Conference on Communications and Networking (BlackSeaCom), Varna, Bulgaria, pp. 1-5, June 2016.

[10] A. Baumgartner, V.S. Reddy, T. Bauschert, "Mobile core network virtualization: A model for combined virtual core network function placement and topology optimization," 2015 1st IEEE Conference on Network Softwari-zation (NetSoft), London, pp. 1-9, 2015.

[11] R. Mijumbi, J. Serrat, J.-L. Gorricho, J. Rubio-Loyola, S. Davy, "Server placement and assignment in virtualized radio access networks," 2015 11th International Conference on Network and Service Management (CNSM), Barcelona, pp. 398-401, 2015. 